\title{
A TYPE OF PENDULUM CHRONOSCOPE AND ATTENTION APPARATUS.
}

\author{
BY PROFESSOR JOHN A. BERGSTROM.
}

Of the pendulum chronoscopes which have been constructed for psychological purposes successively by Professors Sanford, ${ }^{1}$ Fitz, ${ }^{2}$ Scripture ${ }^{3}$ and Seashore, ${ }^{4}$ the one here described was more especially suggested by the Fitz-Scripture type. Like this it secures the registration of the intervals by the mechanism of a heavy pendulum carrying a light index, which may be clamped at any point of a scale graduated into hundredths to thousandths of a second.

Aside from the circular mercury cup attachment and a few details of construction, it differs from it chiefly in the mode of carrying and clamping the index. The mechanism of this part is exhibited in cross section in figure $I$, and in a front view in figure II. The same parts in the two figures are referred to by the same letters.

The steel axis $X^{1} X^{2} X^{3}$ is cut so as to leave the solid sectors $K^{1}$ and $K^{2}$, which have an angle of about $30^{\circ}$, in line and with knife edges coincident with the longitudinal center of the axis. These knife edges rest in shallow $\mathrm{V}$-shaped grooves of hardened steel on the vertical supports $V^{1}, V^{2}$, and the parts are so proportioned as to allow the pendulum to swing through the necessary arc without jolting. The axis, which rotates as it would on any other centered bearings, is cut down to a smaller diameter in the forward projecting section $X^{1}$ and carries there

1 E. C. Sanford, A New Pendulum Chronograph, Am. Journal of Pychology, Vol. V., No. 3, April, 1892. Also, The Vernier Chronoscope, Vol. IX., Jen., 1898. 1895 .

q. W. Fitz, A Location Reaction Apparatus. PSYCHOLOGICAL REVIIW, 1895 .

21. W. Scripture, Studies from Yale Psychological Laboratory, Vol. III.,

C. E. Seashore, Spark Chronoscope, University of Iowa Studies in Psychology, Vol. II., 1899. 
the essential parts of the recording mechanism. This consists of the circular armature $A A$, which may slide forward and

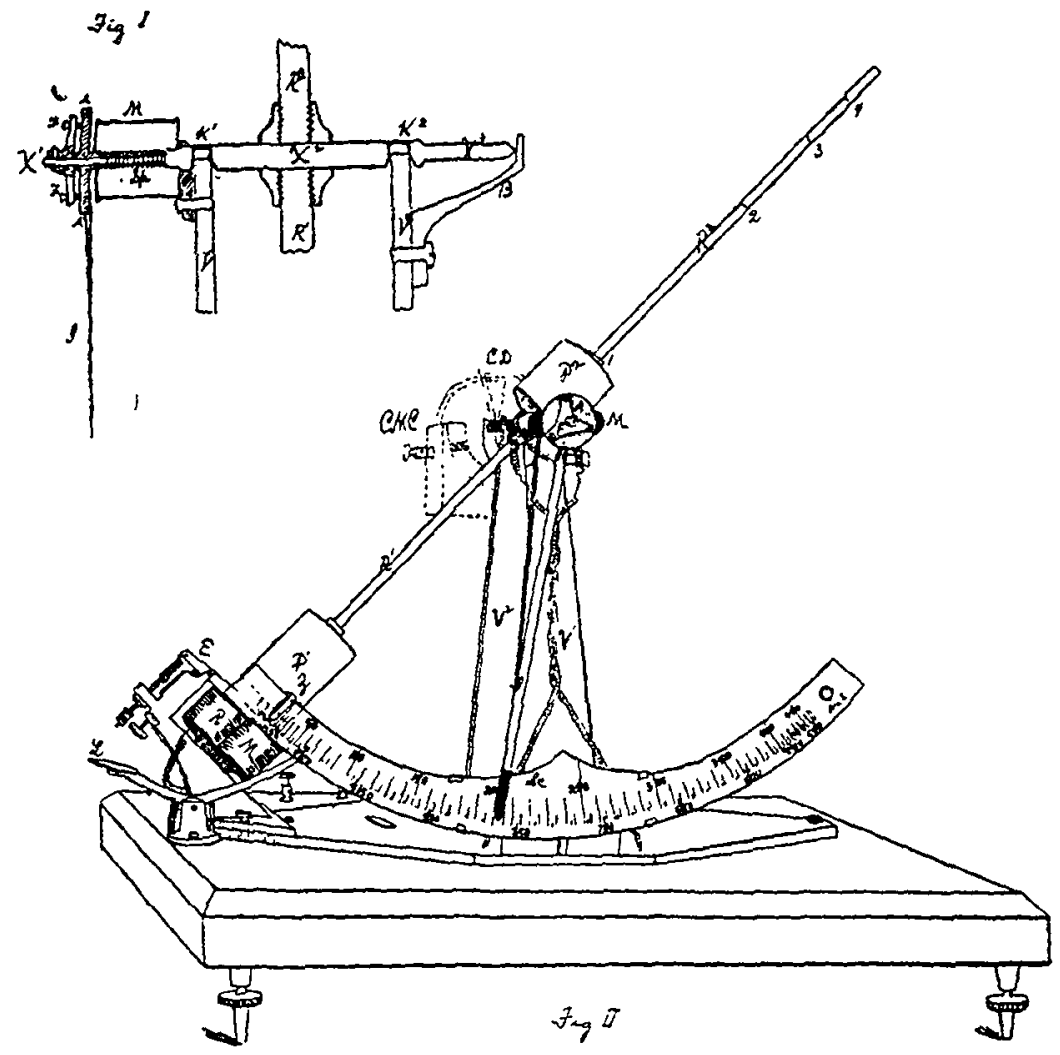

backward or rotate freely on the axis $X^{1}$ and has the index $I$ attached to its circumference. The spiral spring $S p$ holds it against the tripod $F$, which serves to adjust it to the proper distance from the electromagnet $M$ and to provide the friction necessary to carry the index along with the pendulum as it swings.

If a current of sufficient strength is sent through $M$, the circular armature $A$ is at once attracted and moves from the friction tripod $F$ to the magnet, by which it is held immovable as long as may be desired - the pendulum, in the meantime, swinging on with little resistance till it is caught on its return by one of the lower teeth of the release armature $E$. The 
backward pressure exerted by the spring $S p$ on the axis when the armature $A A$ is thus clamped is checked by the brace $B$. The lever $L$ serves to move the pendulum up to its starting point, while the index is set back to zero by moving it against the projecting pin $Z$.

The time of a process is measured by sending one current through the release magnet $R M$ at its beginning; and another through the index magnet $M$ at its end. Both the release of the pendulum and the clamping of the index armature are thus brought about by the closing of the circuits; and the currents may always be kept in the same direction, so that there is no danger of any error from residual magnetism.

This, of course, does not necessitate the use of make-circuit keys in experimentation, but break-circuit keys may be employed as well, if they are used as shunts in the circuits of the magnets. The latter method should, in fact, be adopted in all work requiring a high degree of accuracy on account of the somewhat variable lengthening of the time, due to make-circuit keys. Break-circuit keys, however, while less objectionable, are not wholly so, as variations may be introduced by different tensions of the spring acting on the lever, and the rate and force of the movement of the arm.

In constructing the scales, use was made of the well-known spark method for registering intervals. A fine wire was run down the index so as to leave a point projecting at its end to within a millimeter or less of the metal plate on which the scale is fastened. The front surface of the metal plate was then covered with smoked paper moistened so as to adhere closely, and the sparks from an induction coil interrupted by a hundredvibration tuning fork with a mercury contact were allowed to run over it as the pendulum swung from one end to the other.

Since the pendulum might start at any point between two sparks, successive spark tracings would not coincide, and the number of sparks up to any point on the scale would not, except by chance, represent the absolute time. Moreover, it would not be possible to construct the scales for the return swing, of which use is made in this instrument, on account of the confusion of records. To obviate the trouble and secure the ad- 
vantage of successive coincident tracings, five or six of which were imprinted on the copies for the scales made here, the starting of the pendulum was made dependent upon the dipping into the mercury of the same vibrating point of the tuning fork that broke the circuit of the induction coil for the sparks. By reducing the depth of the dip into the mercury the time between the making of the circuit which releases the pendulum and the breaking of the circuit which gives the spark may conveniently be made about two thousandths of a second, an interval for which compensation can easily be made by the mechanism of releasing the pendulum and clamping the index so that the instrument will register the absolute time.

When the forward scale has become well fixed by half a dozen tracings and has besides been marked, the return scale can be constructed without confusion.

Relative variations in the strength of the currents operating the two magnets produce variations in the records. The instrument must therefore be adjusted for a certain ratio, and the most convenient is, perhaps, that of equality. If the currents are equal, considerable differences in the absolute strength, as from one-half to one and one-half ampères, do not seem to affect the record appreciably, though a current of one ampère is usually employed.

The chronoscope has four scales numbered from one to four and corresponding with times of vibration of about one, one and one-half, two and three seconds. The different times of vibration are produced by placing the counterpoise $P^{2}$ at points $1,2,3$ and 4 on the rod $R^{2}$. For the usual experiments upon the duration of mental process, scales $I$ and 2 or 4 have been found most serviceable. With 4 the forward scale is usually sufficient for the longer reactions, but the return scale of number 2 by giving greater distances for the intervals can be read with greater precision and may be used for the same purpose, and there is less difficulty in securing the proper adjustment of the counterpoise. The counterpoise is eccentric with respect to $R^{2}$ so as to make it possible to give the pendulum the proper balance in each case; and upon this and the proper leveling of the base depends the securing of the absolute time. 
For regulating and learning how to manipulate the instrument use was made of a falling ball apparatus, like one described several years ago by Professor Jastrow. In this, an iron ball about three-fourths of an inch in diameter is held suspended by a short, straight electro-magnet. On the interruption of the current the ball falls and is made to strike a break-circuit key, which may be placed at different distances below the magnet. This break-circuit key serves as a shunt in the circuit of the index magnet, so that opening it leads to the clamping of the index. The time required by the ball to fall to the key is then compared with the readings of the chronoscope. To make the release of the ball and the starting of the pendulum simultaneous presents considerable difficulty, aside from that due to the inertia of the magnet; and two kinds of keys were used for the purpose, one like that constructed by Professor Jastrow with double levers, in which the striking of the first upon the second makes one current, while the lifting of the second from the impact of the first breaks another, and a second in which two currents were broken by the lifting of the same long lever from two insulated points of contact. The second approximates most closely to simultaneity, as the intervals recorded are about .oor' longer when the first is used, and the mechanism is such that the circuit for the release of the pendulum is made first in the double lever key.

The manipulation of these keys represents one of the chief sources of error, as small differences could be produced by varying the rapidity of release.

At points in the scale where the thousandth of a second was represented by about a millimeter, and the scale was so marked, the readings in tens of thousandths being estimated, the writer found that the probable errors of single records deduced from groups of fifty successive records each were for intervals of about .200 and $.300^{\circ}, \pm .00021$ and \pm .00012 respectively. The difference in the probable errors is perhaps due to improvement in manipulation, as the two groups of records were taken in succession, though small errors in the estimation of the ten-thousandths must occur. The corresponding average

'Jastrow, American Joumal of Psychology, Vol. IV., p. 208. 
variations are, $.000168^{8}$ and $.000119^{\circ}$, and the average variation of the instrument for the two series together is .0001 $44^{\circ}$.

In comparison, the average variation of the Hippchronoscope tested by Professor Jastrow by the same method was $.002^{8}$, or nearly fourteen times as great; and Professor Sanford gives $.002^{8}$ as the lowest average variation of the pendulum chronograph.

The accuracy of the other instruments is not stated definitely. Professor Scripture says that the average error of the chronoscope constructed at Yale is not more than $.002^{8}$, and Professor Seashore, that the value of the average deflection in the striking of the spark in the spark-chronoscope amounts to less than $\pm .00 I^{8}$ when the divisions into hundredths equal $5 \mathrm{~mm}$., and varies inversely with the lengths of these divisions.

I am indebted to a group of three students for the following probable errors for single records from groups of fifty records each for the intervals .100, .200, .300 and $.400^{8}$ with scale I., namely, $\pm .00014, \pm .00016, \pm .00022$ and $\pm .00015^{8}$ respectively; and for intervals .200 and .400 with scale II., \pm .00013 and $\pm .00024^{\prime \prime}$, the last group being taken hastily at the end of the period. In this case the estimate into ten-thousandths is, perhaps, less certain, because it was done by the deviation of the point from the center of the dividing lines on the scales, while the scales were marked into only two-hundredths. The correspondence with the previous records, however, is sufficiently close.

Another feature of the instrument is represented by the circular mercury cups $C M C$, and the circular dips $C D$, which are only attached for certain purposes and are consequently represented in dotted outlines. The dips $C D$ can be moved around the axis $X^{3}$ so as to make or break a circuit at any point of the swing of the pendulum, or to make or interrupt a circuit for different intervals of time. For the measurement of intervals less than .050' they may be used to transfer the readings to the central part of the scale, but they come especially into play when the instrument is used for the study of attention to simultaneous stimulation of vision and other senses. As in Wundt's ${ }^{1}$

' Wundt, Physiologische Psychologie, 4th Ed., Vol. II., p. 405. 
apparatus, the moving index supplies one stimulus while the others are brought about by electrical means on the closing of the circuit through the mercury cups. The time errors can be determined directly from the scales, though for extensive experiments more scales than the instrument now possesses would be needed.

The use of the mercury contacts is in some respects inconvenient. Without constant care the accumulation of mercurous oxide and other materials, and to some extent the lack of constancy in the surfaces, particularly on breaking contact, will give rise to large variations in the records, and even with fair care the probable errors of the instrument are two or three times as great as when they are not employed. Thus for breaking circuit with the mercury covered with a layer of either alcohol or kerosene, the probable error for a single record in a group of fifty was $\pm .0006^{s}$. The addition of the alcohol or kerosene makes the circuit break three or four thousandths of a second more quickly. The contacts were cleaned at the beginning and again at the middle of the series. The probable error in the case of the making of the circuit was $\pm .00039^{\mathrm{a}}$. The surfaces and dips were cleaned at the beginning and four times throughout the series, but no alcohol or kerosene was used. The variation in the latter case is due not so much to irregularity as to the fact that from one cleaning of the contacts to another there is a progressive lengthening of the record. The average of this group would therefore be quite reliable as the starting point for the measurement of intervals, and a rather high degree of accuracy might be attained by making due allowance in other cases.

The instrument was constructed about three years ago in the workshop of the psychological laboratory of Indiana University, and has proved very convenient. It can be operated more quickly than it is profitable to make the usual experiments. 